\title{
Existence and regularity of periodic solutions for neutral evolution equations with delays
}

Qiang $\mathrm{Li}^{1 *}$ (D) and Huanhuan Zhang ${ }^{2}$

\section{"Correspondence:}

Iznwnuliqiang@126.com

'Department of Mathematics,

Shanxi Normal University, Linfen,

People's Republic of China

Full list of author information is

available at the end of the article

\section{Springer}

\begin{abstract}
The aim of this paper is to study the periodic problem for neutral evolution equation

$$
(u(t)-G(t, u(t-\xi)))^{\prime}+A u(t)=F(t, u(t), u(t-\tau)), \quad t \in \mathbb{R},
$$

in a Banach space $X$, where $A: D(A) \subset X \rightarrow X$ is a closed linear operator, and $-A$ generates a compact analytic operator semigroup $T(t)(t \geq 0)$. With the aid of the analytic operator semigroup theories and some fixed point theorems, we obtain the existence and uniqueness of periodic mild solution for the neutral evolution equation. The regularity of periodic mild solutions for the evolution equation with delay is studied, and some existence results of the classical and strong solutions are obtained. In the end, we give an example to illustrate the applicability of abstract results. Our works greatly improve and generalize the relevant results of existing literature.
\end{abstract}

MSC: 34K30; 34K40; 47D03; 35A09

Keywords: Evolution equation with delay; Mild solutions; Strong solution; Compact analytic semigroup; Fixed point theorem

\section{Introduction}

Let $X$ be a real Banach space with norm $\|\cdot\|$. The purpose of this paper is to discuss the existence and regularity of $\omega$-periodic solutions for the abstract neutral functional differential equation with delays

$$
(u(t)-G(t, u(t-\xi)))^{\prime}+A u(t)=F(t, u(t), u(t-\tau)), \quad t \in \mathbb{R}
$$

in $X$, where $A: D(A) \subset X \rightarrow X$ is a closed linear operator, and $-A$ generates a compact and exponentially stable analytic operator semigroup $T(t)(t \geq 0)$ on $X, G, F$ are appropriate continuous functions which will be specified later, $\xi$, $\tau$ are positive constants which denote the time delays.

The theory of partial differential equations with delays has extensive physical background and realistic mathematical model, and it has undergone a rapid development in the last fifty years, see $[19,42]$ and the references therein. During the last few decades, more researchers have given special attention to the study of the equation, in which the delay argument occurs in the derivative of the state variable as well as in the independent variable, so-called neutral differential equations.

(c) The Author(s) 2019. This article is distributed under the terms of the Creative Commons Attribution 4.0 International License (http://creativecommons.org/licenses/by/4.0/), which permits unrestricted use, distribution, and reproduction in any medium, provided you give appropriate credit to the original author(s) and the source, provide a link to the Creative Commons license, and indicate if changes were made. 
Neutral differential equations have many applications. They can model a lot of problems arising from engineering, such as population dynamics, transmission line, immune response, or distribution of albumin in the blood. For instance, in the theory of heat conduction in fading memory material, see $[9,37]$, the following partial neutral differential equation

$$
\frac{d}{d t}\left(u(t, x)+\int_{-\infty}^{t} k_{1}(t-s) u(s, x) d s\right)-c \Delta u(t, x)=\int_{-\infty}^{t} k_{2}(t-s) \Delta u(s, x) d s
$$

has been frequently used to describe these phenomena, which has better effects than partial differential equations without neutral item, where $\Omega \subset \mathbb{R}^{n}$ is a bounded domain with a sufficiently smooth boundary $\partial \Omega,(t, x) \in[0, \infty) \times \Omega$ represents the temperature in $x$ at the time $t, c$ is a physical constant, $k_{1}, k_{2}: \mathbb{R} \rightarrow \mathbb{R}$ are the internal energy and the heat flux relaxation respectively. If the solution $u$ is known on $(-\infty, 0], k_{1} \equiv 0$ on $(r, \infty)$, and $k_{2} \equiv 0$, then we can transform system (1.2) into the abstract neutral evolution equation (1.1).

In fact, many of partial neutral differential equations can be written as first-order abstract neutral functional differential equations on an appropriate Banach space. There has been an increasing interest in the study of the abstract neutral evolution equations of the form (1.1). The existence and uniqueness of mild solutions to the abstract neutral evolution equations with delay have been considered by many authors in literature. Here we only mention $[1-3,13,18,20,22,23]$.

It is noteworthy at this point that the problem concerning periodic solutions of partial neutral functional differential equations has become an important area of investigation. The periodic problems can take into account seasonal fluctuations occurring in the phenomena appearing in the models, and have been studied by some researchers in recent years. Specially, the existence of periodic solutions for the neutral evolution equations has been considered by several authors, see [4-7, 11, 12, 14-17, 21, 24, 43] and the references therein for more comments. We notice that, in many works, the key assumption of prior boundedness is employed and the most important ingredient to prove the existence of periodic solutions is to show that Poincaré's mapping is condensing. Thus, a fixed point theorem can be used to derive periodic solutions. For the delayed evolution equations without neutral item, the existence of periodic solutions has been discussed by more authors, see $[8,26,30-32,34-36,44]$ and the references therein.

Although there have been many meaningful results on the neutral evolution equation periodic problem in a Banach space, to our knowledge, these results have a relatively large limitation. First of all, the most popular approach is the use of boundedness or ultimate boundedness of solutions and the compactness of Poincaré map realized through some compact embeddings. However, in some concrete applications, it is difficult to choose appropriate initial conditions to guarantee the boundedness of the solution. Secondly, we observe that the most popular condition imposed on the nonlinear term $F$ is its Lipschitztype condition. In fact, for equations arising in complicated reaction-diffusion processes, the nonlinear function $F$ represents the source of material or population, which depends on time in diversified manners in many contexts. Thus, we may not hope to have the Lipschitz-type condition of $F$. Finally, there are few papers to study the regularity of periodic solutions for the neutral evolution equation with delays. 
Motivated by the papers mentioned above, we aim in this work to study the existence and regularity of periodic solutions for the partial neutral functional differential equation (1.1). In this paper, it is worth mentioning that the assumption of prior boundedness of solutions is not necessary. More precisely, the nonlinear term $F$ only satisfies some growth conditions and the functions $G$ and $F$ may not be defined on the whole space $X$. These conditions are much weaker than Lipschitz conditions. The obtained results will improve the main results in $[6,14,21]$ and develop the work in [30].

The paper is organized as follows. In Sect. 2, we collect some known notions and results on the fractional powers of the generator of an analytic semigroup and provide preliminary results to be used in theorems stated and proved in the paper. In Sect. 3, we apply the operator semigroup theory to find the periodic mild solutions for Eq. (1.1), and in Sect. 4, we investigate conditions for Eq. (1.1) to have the classical and strong periodic solutions. In the last section, we give an example to illustrate the applicability of abstract results obtained in Sect. 3 and Sect. 4.

\section{Preliminaries}

Throughout this paper, we assume that $X$ is a Banach space with norm $\|\cdot\|$.

Now, we recall some notions and properties of operator semigroups, which are essential for us. Assume that $A: D(A) \subset X \rightarrow X$ is a closed linear operator and $-A$ generates a compact analytic operator semigroup $T(t)(t \geq 0)$ on $X$. For the detailed theory of operator semigroups, we refer to [38].

For a general $C_{0}$-semigroup $T(t)(t \geq 0)$, there exist $M \geq 1$ and $v \in \mathbb{R}$ such that $\|T(t)\| \leq$ $M e^{v t}$ for all $t \geq 0$ (see [38]). Let

$$
v_{0}=\inf \left\{\gamma \in \mathbb{R} \mid \text { There exists } M \geq 1 \text { such that }\|T(t)\| \leq M e^{v t}, \forall t \geq 0\right\},
$$

then $v_{0}$ is called the growth exponent of the semigroup $T(t)(t \geq 0)$. Specially, if $v_{0}<0$, then $T(t)(t \geq 0)$ is called an exponentially stable $C_{0}$-semigroup. Furthermore, $v_{0}$ can be also obtained by the formula $v_{0}=\lim \sup _{t \rightarrow+\infty} \frac{\ln \|T(t)\|}{t}$. If $T(t)$ is continuous in the uniform operator topology for every $t>0$ in $X$, it is well known that $v_{0}$ can also be determined by $\nu_{0}=-\inf \{\operatorname{Re} \lambda \mid \lambda \in \sigma(A)\}$ (see $[38,41]$ ). We know that $T(t)(t \geq 0)$ is continuous in the uniform operator topology for $t>0$ if $T(t)(t \geq 0)$ is a compact semigroup or an analytic semigroup (see [25]).

If $T(t)(t \geq 0)$ is analytic satisfying $0 \in \rho(A)(\rho(A)$ is the resolvent set of $A)$, then for any $\alpha>0$, we can define $A^{-\alpha}$ by

$$
A^{-\alpha}:=\frac{1}{\Gamma(\alpha)} \int_{0}^{\infty} t^{\alpha-1} T(t) d t
$$

It follows that each $A^{-\alpha}$ is an injective continuous endomorphism of $X$. Hence we can define $A^{\alpha}:=\left(A^{-\alpha}\right)^{-1}$, which is a closed bijective linear operator on $X$. We define $D\left(A^{\alpha}\right)$ as the domain of the operator $A^{\alpha}$, obviously, the subspace $D\left(A^{\alpha}\right)$ is dense in $X$. Furthermore, $D\left(A^{\alpha}\right)$ endowed with the norm $\|x\|_{\alpha}:=\left\|A^{\alpha} x\right\|$ for all $x \in D\left(A^{\alpha}\right)$ is a Banach space. Next, we denote $D\left(A^{\alpha}\right)$ by $X_{\alpha}$ and $C_{\alpha}:=\left\|A^{-\alpha}\right\|$. The following properties are well known [38].

Lemma 2.1 If $T(t)(t \geq 0)$ is an analytic semigroup with infinitesimal generator A satisfying $0 \in \rho(A)$, then 
(i) $D\left(A^{\alpha}\right)$ is a Banach space for $0 \leq \alpha \leq 1$;

(ii) $A^{-\alpha}$ is a bounded linear operator for $0 \leq \alpha \leq 1$ in $X$;

(iii) $T(t): X \rightarrow D\left(A^{\alpha}\right)$ for each $t>0$;

(iv) $A^{\alpha} T(t) x=T(t) A^{\alpha} x$ for each $x \in D\left(A^{\alpha}\right)$ and $t \geq 0$;

(v) for every $t>0, A^{\alpha} T(t)$ is bounded in $X$ and there exists $M_{\alpha}>0$ such that

$$
\left\|A^{\alpha} T(t)\right\| \leq M_{\alpha} t^{-\alpha}
$$

moreover, if $\alpha \in(0,1)$, then $M_{\alpha}=M \Gamma(\alpha)$;

(vi) $X_{\beta} \hookrightarrow X_{\alpha}$ for $0 \leq \alpha \leq \beta \leq 1$ (with $X_{0}=X$ and $X_{1}=D(A)$ ). $X_{\beta} \hookrightarrow X_{\alpha}$ is compact whenever the resolvent operator of $A$ is compact.

Observe by Lemma 2.1(iii) and (iv) that the restriction $T_{\alpha}(t)$ of $T(t)$ to $X_{\alpha}$ is exactly the part of $T(t)$ in $X_{\alpha}$. Moreover, for any $x \in X_{\alpha}$, we have

$$
\left\|T_{\alpha}(t) x\right\|_{\alpha}=\left\|A^{\alpha} T(t) x\right\|=\left\|T(t) A^{\alpha} x\right\| \leq\|T(t)\| \cdot\left\|A^{\alpha} x\right\|=\|T(t)\|\|x\|_{\alpha}
$$

and

$$
\left\|T_{\alpha}(t) x-x\right\|_{\alpha}=\left\|A^{\alpha} T(t) x-A^{\alpha} x\right\|=\left\|T(t) A^{\alpha} x-A^{\alpha} x\right\| \rightarrow 0, \quad t \rightarrow 0 .
$$

It follows that $T_{\alpha}(t)(t \geq 0)$ is a strongly continuous semigroup on $X_{\alpha}$ and $\left\|T_{\alpha}(t)\right\|_{\alpha} \leq$ $\|T(t)\|$ for all $t \geq 0$. To prove our main results, we need the following lemmas.

Lemma 2.2 ([33]) If $T(t)(t \geq 0)$ is a compact semigroup in $X$, then $T_{\alpha}(t)(t \geq 0)$ is a compact semigroup in $X_{\alpha}$.

Lemma 2.3 ([10]) If $X$ is reflexive, then $X_{\alpha}$ is also reflexive.

Now, recall some basic facts on abstract linear evolution equations, which are needed to prove our main results.

Let $J$ denote the infinite interval $[0, \infty)$ and $h: J \rightarrow X$, consider the initial value problem of the linear evolution equation

$$
\left\{\begin{array}{l}
u^{\prime}(t)+A u(t)=h(t), \quad t \in J \\
u(0)=x_{0}
\end{array}\right.
$$

It is well known, when $x_{0} \in D(A)$ and $h \in C^{1}(J, X)$, the initial value problem (2.1) has a unique classical solution $u \in C^{1}(J, X) \cap C\left(J, X_{1}\right)$ expressed by

$$
u(t)=T(t) x_{0}+\int_{0}^{t} T(t-s) h(s) d s
$$

Generally, for $x_{0}$ and $h \in C(J, X)$, the function $u$ given by (2.2) belongs to $C(J, X)$ and it is called a mild solution of the linear evolution equation (2.1). A function $u: J \rightarrow X$ is called a strong solution of Eq. (2.1) if it is continuous on $J$, differentiable a.e. on $(0, \infty)$, $u^{\prime} \in L_{\mathrm{loc}}^{1}(J, X)$, and satisfies Eq. (2.1). Furthermore, we have the following results. 
Lemma 2.4 ([38]) Let $h \in C([0, a], X)(a>0), 0 \leq \alpha<\beta \leq 1, \mu=\beta-\alpha, x_{0} \in X_{\beta}$, then the mild solution $u$ of Eq. (2.1) satisfies $u \in c^{\mu}\left([0, a], X_{\alpha}\right)$.

Lemma 2.5 ([38]) Let $h \in C^{\mu}([0, a], X)(a>0), 0<\mu<1, x_{0} \in X$. Then the mild solution $u$ of Eq. (2.1) is a classical solution on $[0, a]$.

Let $C_{\omega}(\mathbb{R}, X)$ be the Banach space $\{u \in C(\mathbb{R}, X) \mid u(t)=u(t+\omega), t \in \mathbb{R}\}$ endowed with the norm $\|u\|_{C}=\max _{t \in[0, \omega]}\|u(t)\|$, and $C_{\omega}\left(\mathbb{R}, X_{\alpha}\right)$ be the Banach space $\left\{u \in C\left(\mathbb{R}, X_{\alpha}\right) \mid u(t)=\right.$ $u(t+\omega), t \in \mathbb{R}\}$ endowed with the norm $\|u\|_{C \alpha}=\max _{t \in[0, \omega]}\|u(t)\|_{\alpha}$. Clearly, $C_{\omega}\left(\mathbb{R}, X_{\alpha}\right) \hookrightarrow$ $C_{\omega}(\mathbb{R}, X)$.

Given $h \in C_{\omega}(\mathbb{R}, X)$, we consider the existence of an $\omega$-periodic mild solution of the linear evolution equation

$$
u^{\prime}(t)+A u(t)=h(t), \quad t \in \mathbb{R} .
$$

Lemma 2.6 ([28, 29]) If -A generates an exponentially stable $C_{0}$-semigroup $T(t)(t \geq 0)$ in $X$, then for $h \in C_{\omega}(\mathbb{R}, X)$, the linear evolution equation (2.3) has a unique $\omega$-periodic mild solution $u$, which can be expressed by

$$
u(t)=(I-T(\omega))^{-1} \int_{t-\omega}^{t} T(t-s) h(s) d s:=(P h)(t)
$$

and the solution operator $P: C_{\omega}(\mathbb{R}, X) \rightarrow C_{\omega}(\mathbb{R}, X)$ is a bounded linear operator.

Proof For any $v \in\left(0,\left|v_{0}\right|\right)$, there exists $M>0$ such that

$$
\|T(t)\| \leq M e^{-v t} \leq M, \quad t \geq 0 .
$$

In $X$, define the equivalent norm $|\cdot|$ by

$$
|x|=\sup _{t \geq 0}\left\|e^{v t} T(t) x\right\|
$$

then $\|x\| \leq|x| \leq M\|x\|$. By $|T(t)|$ we denote the norm of $T(t)$ in $(X,|\cdot|)$, then for $t \geq 0$, it is easy to obtain that $|T(t)|<e^{-v t}$. Hence, $(I-T(\omega))$ has a bounded inverse operator

$$
(I-T(\omega))^{-1}=\sum_{n=0}^{\infty} T(n \omega),
$$

and its norm satisfies

$$
\left|(I-T(\omega))^{-1}\right| \leq \frac{1}{1-|T(\omega)|} \leq \frac{1}{1-e^{-\nu \omega}} .
$$

Set

$$
x_{0}=(I-T(\omega))^{-1} \int_{0}^{\omega} T(t-s) h(s) d s:=B h,
$$


then the mild solution $u(t)$ of the linear initial value problem (2.1) given by (2.2) satisfies the periodic boundary condition $u(0)=u(\omega)=x_{0}$. For $t \in \mathbb{R}^{+}$, by (2.2) and the properties of the semigroup $T(t)(t \geq 0)$, we have

$$
\begin{aligned}
u(t+\omega) & =T(t+\omega) u(0)+\int_{0}^{t+\omega} T(t+\omega-s) h(s) d s \\
& =T(t)\left(T(\omega) u(0)+\int_{0}^{\omega} T(\omega-s) h(s) d s\right)+\int_{0}^{t} T(t-s) h(s-\omega) d s \\
& =T(t) u(0)+\int_{0}^{t} T(t-s) h(s) d s=u(t) .
\end{aligned}
$$

Therefore, the $\omega$-periodic extension of $u$ on $\mathbb{R}$ is a unique $\omega$-periodic mild solution of Eq. (2.3). By (2.2) and (2.6), the $\omega$-periodic mild solution can be expressed by

$$
\begin{aligned}
u(t) & =T(t) B(h)+\int_{0}^{t} T(t-s) h(s) d s \\
& =(I-T(\omega))^{-1} \int_{t-\omega}^{t} T(t-s) h(s) d s:=(P h)(t) .
\end{aligned}
$$

Evidently, $P: C_{\omega}(\mathbb{R}, X) \rightarrow C_{\omega}(\mathbb{R}, X)$ is a bounded linear operator. In fact, for every $h \in$ $C_{\omega}(\mathbb{R}, X)$,

$$
\begin{aligned}
\|Q h(t)\| & =\left\|(I-T(\omega))^{-1} \int_{t-\omega}^{t} T(t-s) h(s) d s\right\| \\
& \leq\left\|(I-T(\omega))^{-1}\right\| \cdot \int_{t-\omega}^{t}\|T(t-s)\| d s\|h\|_{C} \\
& \leq C M \omega\|h\|_{C},
\end{aligned}
$$

where $C:=\left\|(I-T(\omega))^{-1}\right\|$, which implies that $P$ is bounded. This completes the proof of Lemma 2.6.

To prove our main results, we also need the following lemma.

Lemma 2.7 ([39]) Assume that $Q$ is a condensing operator on a Banach space X, i.e., $Q$ is continuous and takes bounded sets into bounded sets, and $\alpha(Q(D))<\alpha(D)$ for every bounded set $D$ of $X$ with $\alpha(D)>0$. If $Q(\bar{\Omega}) \subset \bar{\Omega}$ for a convex, closed, and bounded set $\bar{\Omega}$ of $X$, then $Q$ has a fixed point in $\bar{\Omega}$ (where $\alpha(\cdot)$ denotes the Kuratowski measure of noncompactness).

Remark 2.8 It is easy to see that, if $Q=Q_{1}+Q_{2}$ with $Q_{1}$ a completely continuous operator and $Q_{2}$ a contractive map, then $Q$ is a condensing operator on $X$.

\section{Existence of mild solution}

Now, we are in a position to state and prove our main results of this section.

Theorem 3.1 For $\alpha \in[0,1)$, we assume that $G: \mathbb{R} \times X_{\alpha} \rightarrow X_{1}$ and $F: \mathbb{R} \times X_{\alpha}^{2} \rightarrow X$ are continuous functions, and for every $x, x_{0}, x_{1} \in X_{\alpha}, G(t, x), F\left(t, x_{0}, x_{1}\right)$ are $\omega$-periodic in $t$. If the following conditions hold: 
(H1) For any $r>0$, there is a positive value function $h_{r}: \mathbb{R} \rightarrow \mathbb{R}^{+}$such that

$$
\sup _{\left\|x_{0}\right\| \alpha,\left\|x_{1}\right\|_{\alpha}<r}\left\|F\left(t, x_{0}, x_{1}\right)\right\| \leq h_{r}(t), \quad t \in \mathbb{R}
$$

the function $s \mapsto \frac{h_{r}(s)}{(t-s)^{\alpha}}$ belongs to $L_{\mathrm{loc}}\left(\mathbb{R}, \mathbb{R}^{+}\right)$for all $t \in \mathbb{R}$ and there is a positive constant $\gamma>0$ such that

$$
\liminf _{r \rightarrow \infty} \frac{1}{r} \int_{t-\omega}^{t} \frac{h_{r}(s)}{(t-s)^{\alpha}} d s=\gamma<\infty
$$

(H2) $G(t, \theta)=\theta$ for $t \in \mathbb{R}$, and there is a constant $L \geq 0$ such that

$$
\|A G(t, x)-A G(t, y)\| \leq L\|x-y\|_{\alpha}, \quad t \in \mathbb{R}, x, y \in X_{\alpha}
$$

(H3) $C M_{\alpha} \gamma+C_{1-\alpha} L+C M_{\alpha} L \frac{\omega^{1-\alpha}}{1-\alpha}<1$, where $C=\left\|(I-T(\omega))^{-1}\right\|$, then Eq. (1.1) has at least one $\omega$-periodic mild solution $u$.

Proof From the assumption, we know that $G(t, u(t-\xi)) \in D(A)$ for every $u \in C_{\omega}\left(\mathbb{R}, X_{\alpha}\right)$, thus, we can rewrite Eq. (1.1) as follows:

$$
\begin{aligned}
& (u(t)-G(t, u(t-\xi)))^{\prime}+A(u(t)-G(t, u(t-\xi))) \\
& =F(t, u(t), u(t-\tau))-A G(t, u(t-\xi)), \quad t \in \mathbb{R} .
\end{aligned}
$$

For any $r>0$, let

$$
\bar{\Omega}_{r}=\left\{u \in C_{\omega}\left(\mathbb{R}, X_{\alpha}\right) \mid\|u\|_{C \alpha} \leq r\right\}
$$

Note that $\bar{\Omega}_{r}$ is a closed ball in $C_{\omega}\left(\mathbb{R}, X_{\alpha}\right)$ with center $\theta$ and radius $r$. Moreover, by condition $(\mathrm{H} 2)$, it follows that

$$
\begin{aligned}
\|T(t-s) A G(s, u(s-\xi))\|_{\alpha} & =\left\|A^{\alpha} T(t-s) A(G(s, u(s-\xi))-G(s, \theta))\right\| \\
& \leq\left\|A^{\alpha} T(t-s)\right\| \cdot\|A(G(s, u(s-\xi))-G(s, \theta))\| \\
& \leq \frac{M_{\alpha} L}{(t-s)^{\alpha}}\|u(s-\xi)\|_{\alpha}
\end{aligned}
$$

which implies that $s \rightarrow T(t-s) A G(s, u(s-\xi))$ is integrable on $[t-\omega, t]$ for each $u \in \bar{\Omega}_{r}$.

Hence, we can define the operator $Q$ on $C_{\omega}\left(\mathbb{R}, X_{\alpha}\right)$ by

$$
\begin{aligned}
Q u(t):= & (I-T(\omega))^{-1} \int_{t-\omega}^{t} T(t-s) F(s, u(s), u(s-\tau)) d s+G(t, u(t-\xi)) \\
& -(I-T(\omega))^{-1} \int_{t-\omega}^{t} T(t-s) A G(s, u(s-\xi)) d s, \quad t \in \mathbb{R} .
\end{aligned}
$$

From Lemma 2.6, it is sufficient to prove that $Q$ has a fixed point. 
Now, we show that there is a positive constant $r$ such that $Q\left(\bar{\Omega}_{r}\right) \subset \bar{\Omega}_{r}$. If this were not the case, then for any $r>0$, there exist $u_{r} \in \bar{\Omega}_{r}$ and $t_{r} \in \mathbb{R}$ such that $\left\|Q u_{r}\left(t_{r}\right)\right\|_{\alpha}>r$. Thus, we see by $(\mathrm{H} 1)$, (H2), and (H3) that

$$
\begin{aligned}
r< & \left\|Q u_{r}\left(t_{r}\right)\right\|_{\alpha} \\
\leq & \left\|(I-T(\omega))^{-1} \int_{t_{r}-\omega}^{t_{r}} T\left(t_{r}-s\right) F\left(s, u_{r}(s), u_{r}(s-\tau)\right) d s\right\|_{\alpha}+\left\|G\left(t_{r}, u_{r}\left(t_{r}-\xi\right)\right)\right\|_{\alpha} \\
& +\left\|(I-T(\omega))^{-1} \int_{t_{r}-\omega}^{t_{r}} T\left(t_{r}-s\right) A G\left(s, u_{r}(s-\xi)\right) d s\right\|_{\alpha} \\
\leq & \left\|(I-T(\omega))^{-1}\right\| \cdot \int_{t_{r}-\omega}^{t_{r}}\left\|A^{\alpha} T\left(t_{r}-s\right)\right\| \cdot\left\|F\left(s, u_{r}(s), u_{r}(s-\tau)\right)\right\| d s \\
& +\left\|A^{\alpha-1}\left(A G\left(t_{r}, u_{r}\left(t_{r}-\xi\right)\right)-A G\left(t_{r}, \theta\right)\right)\right\| \\
& +\left\|(I-T(\omega))^{-1}\right\| \cdot \int_{t_{r}-\omega}^{t_{r}}\left\|A^{\alpha} T\left(t_{r}-s\right)\right\| \cdot\left\|A G\left(s, u_{r}(s-\xi)\right)-A G(s, \theta)\right\| d s \\
\leq & C M_{\alpha} \int_{t_{r}-\omega}^{t_{r}} \frac{h_{r}(s)}{\left(t_{r}-s\right)^{\alpha}} d s+C_{1-\alpha} L\left\|u_{r}\right\|_{C \alpha}+C M_{\alpha} L \int_{t_{r}-\omega}^{t_{r}} \frac{1}{\left(t_{r}-s\right)^{\alpha}} d s\left\|u_{r}\right\|_{C \alpha} \\
\leq & C M_{\alpha} \int_{t_{r}-\omega}^{t_{r}} \frac{h_{r}(s)}{\left(t_{r}-s\right)^{\alpha}} d s+C_{1-\alpha} L r+C M_{\alpha} L \frac{\omega^{1-\alpha}}{1-\alpha} r .
\end{aligned}
$$

Dividing on both sides by $r$ and taking the lower limit as $r \rightarrow \infty$, we have

$$
C M_{\alpha} L \gamma+C_{1-\alpha} L+C M_{\alpha} L \frac{\omega^{1-\alpha}}{1-\alpha} \geq 1
$$

which contradicts (H3). Hence, there is a positive constant $r$ such that $Q\left(\bar{\Omega}_{r}\right) \subset \bar{\Omega}_{r}$.

In order to show that the operator $Q$ has a fixed point on $\bar{\Omega}_{r}$, we introduce the decomposition $Q=Q_{1}+Q_{2}$, where

$$
\begin{aligned}
& Q_{1} u(t):=(I-T(\omega))^{-1} \int_{t-\omega}^{t} T(t-s) F(s, u(s), u(s-\tau)) d s, \\
& Q_{2} u(t):=G(t, u(t-\xi))-(I-T(\omega))^{-1} \int_{t-\omega}^{t} T(t-s) A G(s, u(s-\xi)) d s .
\end{aligned}
$$

Then we will prove that $Q_{1}$ is a compact operator and $Q_{2}$ is a contraction.

Firstly, we prove that $Q_{1}$ is a compact operator. Let $\left\{u_{n}\right\} \subset \bar{\Omega}_{r}$ with $u_{n} \rightarrow u$ in $\bar{\Omega}_{r}$, then by the continuity of $F$, we have

$$
F\left(t, u_{n}(t), u_{n}(t-\tau)\right) \rightarrow F(t, u(t), u(t-\tau)), \quad n \rightarrow \infty,
$$

for each $t \in \mathbb{R}$. Since $\left\|F\left(t, u_{n}(t), u_{n}(t-\tau)\right)-F(t, u(t), u(t-\tau))\right\| \leq 2 h_{r}(t)$ for all $t \in \mathbb{R}$, then the dominated convergence theorem ensures that

$$
\begin{aligned}
& \left\|Q_{1} u_{n}(t)-Q_{1} u(t)\right\|_{\alpha} \\
& \quad=\left\|(I-T(\omega))^{-1} \int_{t-\omega}^{t} T(t-s) F\left(s, u_{n}(s), u_{n}(s-\tau)\right)-F(s, u(s), u(s-\tau)) d s\right\|_{\alpha}
\end{aligned}
$$




$$
\begin{aligned}
& \leq\left\|(I-T(\omega))^{-1}\right\| \int_{t-\omega}^{t}\left\|A^{\alpha} T(t-s)\right\| \cdot\left\|F\left(s, u_{n}(s), u_{n}(s-\tau)\right)-F(s, u(s), u(s-\tau))\right\| d s \\
& \leq C M_{\alpha} \int_{t-\omega}^{t} \frac{\left\|F\left(s, u_{n}(s), u_{n}(s-\tau)\right)-F(s, u(s), u(s-\tau))\right\|}{(t-s)^{\alpha}} d s \\
& \rightarrow 0 \quad \text { as } n \rightarrow \infty,
\end{aligned}
$$

which implies that $\left\|Q_{1} u_{n}-Q_{1} u\right\|_{C \alpha} \rightarrow 0$ as $n \rightarrow \infty$, i.e., $Q_{1}$ is continuous.

It is easy to see that $Q_{1}$ maps $\bar{\Omega}_{r}$ into a bounded set in $C_{\omega}\left(\mathbb{R}, X_{\alpha}\right)$. Now, we demonstrate that $Q\left(\bar{\Omega}_{r}\right)$ is equicontinuous. For every $u \in \bar{\Omega}_{r}$, by the periodicity of $u$, we only consider it on $[0, \omega]$. Set $0 \leq t_{1}<t_{2} \leq \omega$, we get that

$$
\begin{aligned}
Q_{1} u\left(t_{2}\right)-Q_{1} u\left(t_{1}\right) \\
=(I-T(\omega))^{-1} \int_{t_{2}-\omega}^{t_{2}} T\left(t_{2}-s\right) F(s, u(s), u(s-\tau)) d s \\
\quad-(I-T(\omega))^{-1} \int_{t_{1}-\omega}^{t_{1}} T\left(t_{1}-s\right) F(s, u(s), u(s-\tau)) d s \\
=(I-T(\omega))^{-1} \int_{t_{2}-\omega}^{t_{1}}\left(T\left(t_{2}-s\right)-T\left(t_{1}-s\right)\right) F(s, u(s), u(s-\tau)) d s \\
\quad-(I-T(\omega))^{-1} \int_{t_{1}-\omega}^{t_{2}-\omega} T\left(t_{1}-s\right) F(s, u(s), u(s-\tau)) d s \\
\quad+(I-T(\omega))^{-1} \int_{t_{1}}^{t_{2}} T\left(t_{2}-s\right) F(s, u(s), u(s-\tau)) d s \\
:=
\end{aligned}
$$

It is clear that

$$
\left\|Q_{1} u\left(t_{2}\right)-Q_{1} u\left(t_{1}\right)\right\|_{\alpha} \leq\left\|I_{1}\right\|_{\alpha}+\left\|I_{2}\right\|_{\alpha}+\left\|I_{3}\right\|_{\alpha}
$$

Thus, we only need to check that $\left\|I_{i}\right\|_{\alpha}$ tends to 0 independently of $u \in \bar{\Omega}_{r}$ when $t_{2}-t_{1} \rightarrow 0$, $i=1,2,3$. From the continuity of $t \mapsto\|T(t)\|$ for $t>0$ and condition (H1), we can easily see

$$
\begin{aligned}
\left\|I_{1}\right\|_{\alpha} & \leq C \cdot \int_{t_{2}-\omega}^{t_{1}}\left\|A^{\alpha}\left(T\left(t_{2}-s\right)-T\left(t_{1}-s\right)\right)\right\| \cdot\|F(s, u(s), u(s-\tau))\| d s \\
& \leq C \cdot \int_{t_{2}-\omega}^{t_{1}}\left\|T\left(\frac{t_{2}-s}{2}+\frac{t_{2}-t_{1}}{2}\right)-T\left(\frac{t_{1}-s}{2}\right)\right\| \cdot\left\|A^{\alpha} T\left(\frac{t_{1}-s}{2}\right)\right\| \cdot h_{r}(s) d s \\
& \leq C M_{\alpha} \int_{t_{2}-\omega}^{t_{1}}\left\|T\left(\frac{t_{2}-s}{2}+\frac{t_{2}-t_{1}}{2}\right)-T\left(\frac{t_{1}-s}{2}\right)\right\| \cdot \frac{h_{r}(s)}{\left(\frac{t_{1}-s}{2}\right)^{\alpha}} d s \\
& \rightarrow 0, \quad \text { as } t_{2}-t_{1} \rightarrow 0, \\
\left\|I_{2}\right\|_{\alpha} & \leq C \cdot \int_{t_{1}-\omega}^{t_{2}-\omega}\left\|A T\left(t_{1}-s\right)\right\| \cdot\|F(s, u(s), u(s-\tau))\| d s
\end{aligned}
$$




$$
\begin{aligned}
& \leq C M_{\alpha} \cdot \int_{t_{1}-\omega}^{t_{2}-\omega} \frac{h_{r}(s)}{\left(t_{1}-s\right)^{\alpha}} d s \\
& \rightarrow 0, \quad \text { as } t_{2}-t_{1} \rightarrow 0, \\
\left\|I_{3}\right\|_{\alpha} & \left.\leq C \cdot \int_{t_{1}}^{t_{2}}\left\|A\left(T\left(t_{2}-s\right)\right)\right\| \cdot \| F(s, u(s), u(s-\tau))\right) \| d s \\
& \leq C M_{\alpha} \cdot \int_{t_{1}}^{t_{2}} \frac{h_{r}(s)}{\left(t_{2}-s\right)^{\alpha}} d s \\
& \rightarrow 0, \quad \text { as } t_{2}-t_{1} \rightarrow 0 .
\end{aligned}
$$

As a result, $\left\|Q_{1} u\left(t_{2}\right)-Q_{1} u\left(t_{1}\right)\right\|_{\alpha}$ tends to 0 independently of $u \in \bar{\Omega}_{r}$ as $t_{2}-t_{1} \rightarrow 0$, which means that $Q_{1}\left(\bar{\Omega}_{r}\right)$ is equicontinuous.

It remains to show that $\left(Q_{1} \bar{\Omega}_{r}\right)(t)$ is relatively compact in $X_{\alpha}$ for all $t \in \mathbb{R}$. To do this, we define a set $\left(Q_{\varepsilon} \bar{\Omega}_{r}\right)(t)$ by

$$
\left(Q_{\varepsilon} \bar{\Omega}_{r}\right)(t):=\left\{\left(Q_{\varepsilon} u\right)(t) \mid u \in \bar{\Omega}_{r}, 0<\varepsilon<\omega, t \in \mathbb{R}\right\}
$$

where

$$
\begin{aligned}
\left(Q_{\varepsilon} u\right)(t) & =(I-T(\omega))^{-1} \int_{t-\omega}^{t-\varepsilon} T_{\alpha}(t-s) F(s, u(s), u(s-\tau)) d s \\
& =T_{\alpha}(\varepsilon)(I-T(\omega))^{-1} \int_{t-\omega}^{t-\varepsilon} T_{\alpha}(t-s-\varepsilon) F(s, u(s), u(s-\tau)) d s .
\end{aligned}
$$

From Lemma 2.2, the operator $T_{\alpha}(\varepsilon)$ is compact in $X_{\alpha}$, it follows that the set $\left(Q_{\varepsilon} \bar{\Omega}_{r}\right)(t)$ is relatively compact in $X_{\alpha}$. For any $u \in \bar{\Omega}_{r}$ and $t \in \mathbb{R}$, from the following inequality

$$
\begin{aligned}
& \left\|Q_{1} u(t)-Q_{\varepsilon} u(t)\right\|_{\alpha} \\
& \quad \leq C \int_{t-\varepsilon}^{t}\left\|T_{\alpha}(t-s) F(s, u(s), u(s-\tau))\right\|_{\alpha} d s \\
& \leq C \int_{t-\varepsilon}^{t}\left\|A^{\alpha} T(t-s) F(s, u(s), u(s-\tau))\right\| d s \\
& \leq C M_{\alpha} \int_{t-\varepsilon}^{t} \frac{h_{r}(s)}{(t-s)^{\alpha}} d s,
\end{aligned}
$$

one can obtain that the set $\left(Q_{1} \bar{\Omega}_{r}\right)(t)$ is relatively compact in $X_{\alpha}$ for all $t \in \mathbb{R}$.

Thus, the Arzela-Ascoli theorem guarantees that $Q_{1}$ is a compact operator.

Secondly, we prove that $Q_{2}$ is a contraction. Let $u, v \in \bar{\Omega}_{r}$, by condition (H2), Lemma 2.1(vi), and Lemma 2.6, we have

$$
\begin{aligned}
\left\|Q_{2} u(t)-Q_{2} v(t)\right\|_{\alpha} & \\
= & \| G(t, u(t-\xi))-(I-T(\omega))^{-1} \int_{t}^{t-\omega} T(t-s) A G(s, u(s-\xi)) d s \\
& \quad-G(t, v(t-\xi))+(I-T(\omega))^{-1} \int_{t}^{t-\omega} T(t-s) A G(s, v(s-\xi)) \|_{\alpha} \\
\leq & \|G(t, u(t-\xi))-G(t, v(t-\xi))\|_{\alpha}
\end{aligned}
$$




$$
\begin{aligned}
& +\left\|(I-T(\omega))^{-1} \int_{t}^{t-\omega} T(t-s) A(G(s, u(s-\xi))-G(s, v(s-\xi))) d s\right\|_{\alpha} \\
\leq & \| A^{\alpha-1}(A G(t, u(t-\xi))-A G(t, v(t-\xi)) \| \\
& +\left\|(I-T(\omega))^{-1}\right\| \cdot \int_{t}^{t-\omega}\|A T(t-s)\|\|A(G(s, u(s-\xi))-G(s, v(s-\xi)))\| d s \\
\leq & C_{1-\alpha} \| A G(t, u(t-\xi)-A G(t, v(t-\delta)) \| \\
& +C M \cdot \int_{t}^{t-\omega}\|A G(s, u(s-\xi))-A G(s, v(s-\xi))\| d s \\
\leq & C_{1-\alpha} L\|u(t-\xi)-v(t-\xi)\|_{\alpha}+C M_{\alpha} L \int_{t}^{t-\omega} \frac{1}{(t-s)^{\alpha}}\|u(s-\xi)-v(s-\xi)\|_{\alpha} d s \\
\leq & \left(C_{1-\alpha} L+C M_{\alpha} L \frac{\omega^{1-\alpha}}{1-\alpha}\right)\|u-v\|_{C \alpha} ;
\end{aligned}
$$

therefore,

$$
\left\|Q_{2} u-Q_{2} v\right\|_{C} \leq\left(C_{1-\alpha} L+C M_{\alpha} L \frac{\omega^{1-\alpha}}{1-\alpha}\right)\|u-v\|_{C \alpha} .
$$

Since $C M_{\alpha} L \gamma+C_{1-\alpha} L+C M_{\alpha} L \frac{\omega^{1-\alpha}}{1-\alpha}<1$, so $C_{1-\alpha} L+C M_{\alpha} L \frac{\omega^{1-\alpha}}{1-\alpha}<1$, it follows that $Q_{2}$ is a contraction.

By Lemma 2.7, we know that $Q$ has a fixed point $u \in \bar{\Omega}_{r}$, that is, Eq. (1.1) has a $\omega$-periodic mild solution. The proof is completed.

In condition (H1), if the function $h_{r}$ is independent of $t$, we can easily obtain a constant $\gamma \geq 0$ satisfying (H3). For example, we replace condition (H1) with the following: (H1') There are positive constants $a_{0}, a_{1}$, and $K$ such that

$$
\left\|F\left(t, x_{0}, x_{1}\right)\right\| \leq a_{0}\left\|x_{0}\right\|_{\alpha}+a_{1}\left\|x_{1}\right\|_{\alpha}+K
$$

for $t \in \mathbb{R}$ and $x_{0}, x_{1} \in X_{\alpha}$.

In this case, for any $r>0$ and $x_{0}, x_{1} \in X_{\alpha}$ with $\left\|x_{0}\right\|_{\alpha},\left\|x_{1}\right\|_{\alpha} \leq r$, we have

$$
\left\|F\left(t, x_{0}, x_{1}\right)\right\| \leq r\left(a_{0}+a_{1}\right)+K:=h_{r}(t), \quad t \in \mathbb{R},
$$

thus,

$$
\liminf _{r \rightarrow \infty} \frac{1}{r} \int_{t-\omega}^{t} \frac{h_{r}(s)}{(t-s)^{\alpha}} d s=\left(a_{0}+a_{1}\right) \frac{\omega^{1-\alpha}}{1-\alpha}:=\gamma>0 .
$$

Therefore, we have the following result.

Corollary 3.2 For $\alpha \in[0,1)$, we assume that $G: \mathbb{R} \times X_{\alpha} \rightarrow X_{1}$ and $F: \mathbb{R} \times X_{\alpha}^{2} \rightarrow X$ are continuous functions, and for every $x, x_{0}, x_{1} \in X_{\alpha}, G(t, x), F\left(t, x_{0}, x_{1}\right)$ are $\omega$-periodic in $t$. If conditions $\left(\mathrm{H}^{\prime}\right),(\mathrm{H} 2)$, and

$\left(\mathrm{H}^{\prime}\right) C M_{\alpha}\left(a_{0}+a_{1}+L\right) \frac{\omega^{1-\alpha}}{1-\alpha}+C_{1-\alpha} L<1$, where $C=\left\|(I-T(\omega))^{-1}\right\|$, hold, then Eq. (1.1) has at least one $\omega$-periodic mild solution $u$. 
Furthermore, we assume that $F$ satisfies the Lipschitz condition, namely

$\left(\mathrm{H} 1^{\prime \prime}\right)$ There are positive constants $a_{0}, a_{1}$ such that

$$
\left\|F\left(t, x_{0}, x_{1}\right)-F\left(t, y_{0}, y_{1}\right)\right\| \leq a_{0}\left\|x_{0}-y_{0}\right\|_{\alpha}+a_{1}\left\|x_{1}-y_{1}\right\|_{\alpha}, \quad t \in \mathbb{R}, x_{0}, x_{1}, y_{0}, y_{1} \in X_{\alpha},
$$

then we can obtain the following result.

Theorem 3.3 For $\alpha \in[0,1)$, we assume that $G: \mathbb{R} \times X_{\alpha} \rightarrow X_{1}$ and $F: \mathbb{R} \times X_{\alpha}^{2} \rightarrow X$ are continuous functions, and for every $x, x_{0}, x_{1} \in X_{\alpha}, G(t, x), F\left(t, x_{0}, x_{1}\right)$ are $\omega$-periodic in $t$. If conditions (H1"), (H2), and (H3') hold, then Eq. (1.1) has a unique w-periodic mild solution $u$.

Proof From ( $\left.\mathrm{H}^{\prime \prime}\right)$ we easily see that $\left(\mathrm{H}^{\prime}\right)$ holds. In fact, for any $t \in \mathbb{R}$ and $x_{0}, x_{1} \in X_{\alpha}$, by condition $\left(\mathrm{H} 1^{\prime \prime}\right)$,

$$
\begin{aligned}
\left\|F\left(t, x_{0}, x_{1}\right)\right\| & \leq\left\|F\left(t, x_{0}, x_{1}\right)-F(t, \theta, \theta)\right\|+\|F(t, \theta, \theta)\| \\
& \leq a_{0}\left\|x_{0}\right\|_{\alpha}+a_{1}\left\|x_{1}\right\|_{\alpha}+\|F(t, \theta, \theta)\| .
\end{aligned}
$$

From the continuity and periodicity of $F$, we can choose $K=\max _{t \in[0, \omega]}\|F(t, \theta, \theta)\|$, thus, condition (H1') holds. Hence, by Corollary 3.2, Eq. (1.1) has $\omega$-periodic mild solutions. Let $u_{1}, u_{2} \in C_{\omega}\left(\mathbb{R}, X_{\alpha}\right)$ be the $\omega$-periodic mild solutions of Eq. (1.1), then they are the fixed points of the operator $Q$ which is defined by (3.3). Hence,

$$
\begin{aligned}
\left\|Q u_{2}(t)-Q u_{1}(t)\right\|_{\alpha} & \left\|(I-T(\omega))^{-1} \int_{t-\omega}^{t} T(t-s)\left(F\left(s, u_{2}(s), u_{2}(s-\tau)\right)-F\left(s, u_{1}(s), u_{1}(s-\tau)\right)\right) d s\right\|_{\alpha} \\
\leq & +\left\|G\left(t, u_{2}(t-\xi)\right)-G\left(t, u_{1}(t-\xi)\right)\right\|_{\alpha} \\
& +\left\|(I-T(\omega))^{-1} \int_{t-\omega}^{t} T(t-s)\left(A G\left(s, u_{2}(s-\xi)\right)-A G\left(s, u_{1}(s-\xi)\right)\right) d s\right\|_{\alpha} \\
\leq & \left.C \cdot \int_{t-\omega}^{t}\left\|A^{\alpha} T(t-s)\right\| \cdot \| F\left(s, u_{2}(s), u_{2}(s-\tau)\right)-F\left(s, u_{1}(s), u_{1}(s-\tau)\right)\right) \| d s \\
& +\left\|A^{\alpha-1}\left(A G\left(t, u_{2}(t-\xi)\right)-A G\left(t, u_{1}(t-\xi)\right)\right)\right\| \\
& +C \cdot \int_{t-\omega}^{t}\left\|A^{\alpha} T(t-s)\right\| \cdot\left\|A G\left(s, u_{2}(s-\xi)\right)-A G\left(s, u_{1}(s-\xi)\right)\right\| d s \\
\leq & C M_{\alpha} \cdot \int_{t-\omega}^{t} \frac{1}{(t-s)^{\alpha}}\left(a_{0}\left\|u_{2}(s)-u_{1}(s)\right\|_{\alpha}+a_{1}\left\|u_{2}(s-\tau)-u_{1}(s-\tau)\right\|_{\alpha}\right) d s \\
& +C_{1-\alpha} L\left\|u_{2}(t-\xi)-u_{1}(t-\xi)\right\|_{\alpha} \\
& +C M_{\alpha} \cdot \int_{t-\omega}^{t} \frac{L}{(t-s)^{\alpha}}\left\|u_{2}(t-\xi)-u_{1}(t-\xi)\right\|_{\alpha} d s \\
\leq & C M_{\alpha} \frac{\omega^{1-\alpha}}{1-\alpha}\left(a_{0}+a_{1}\right)\left\|u_{2}-u_{1}\right\|_{C \alpha}+C_{1-\alpha} L\left\|u_{2}-u_{1}\right\|_{C \alpha}+C M_{\alpha} \frac{\omega^{1-\alpha}}{1-\alpha} L\left\|u_{2}-u_{1}\right\|_{C \alpha} \\
= & \left(C M_{\alpha}\left(a_{0}+a_{1}+L\right) \frac{\omega^{1-\alpha}}{1-\alpha}+C_{1-\alpha} L\right) \cdot\left\|u_{2}-u_{1}\right\|_{C_{\alpha}},
\end{aligned}
$$


which implies that $\left\|u_{2}-u_{1}\right\|_{C \alpha}=\left\|Q u_{2}-Q u_{1}\right\|_{C \alpha} \leq\left(C M \omega\left(a_{0}+a_{1}+L\right)+C_{1-\alpha} L\right) \cdot \| u_{2}-$ $u_{1} \|_{C \alpha}$. From this and condition (H3'), it follows that $u_{2}=u_{1}$. Thus, Eq. (1.1) has only one $\omega$-periodic mild solution.

\section{Regularity of mild solutions}

In this section, we discuss the regularity properties of the $\omega$-periodic mild solution of Eq. (1.1) and present essential conditions on the nonlinearity $F$ and $G$ to guarantee that Eq. (1.1) has $\omega$-periodic classical and strong solutions.

Now, we are in a position to state and prove the main result of this section.

Theorem 4.1 For $\alpha \in[0,1)$, we assume that $G: \mathbb{R} \times X_{\alpha} \rightarrow X_{1}$ and $F: \mathbb{R} \times X_{\alpha}^{2} \rightarrow X$ are continuous functions, and for every $x, x_{0}, x_{1} \in X_{\alpha}, G(t, x), F\left(t, x_{0}, x_{1}\right)$ are $\omega$-periodic in $t$. If the following conditions hold:

(H4) There exist $L_{1}$ and $\mu_{1} \in(0,1)$ such that

$$
\left\|F\left(t_{2}, x_{0},, x_{1}\right)-F\left(t_{1}, y_{0}, y_{1}\right)\right\| \leq L_{1}\left(\left|t_{2}-t_{1}\right|^{\mu_{1}}+\left\|x_{0}-y_{0}\right\|_{\alpha}+\left\|x_{1}-y_{1}\right\|_{\alpha}\right)
$$

for each $t_{1}, t_{2} \in \mathbb{R}$ and $x_{0}, x_{1}, y_{0}, y_{1} \in X_{\alpha}$

(H5) $G(t, \theta)=\theta$ for $t \in \mathbb{R}$, there exist $L_{2}$ and $\mu_{2} \in(0,1)$ such that

$$
\left\|A G\left(t_{2}, x\right)-A G\left(t_{1}, y\right)\right\| \leq L_{2}\left(\left|t_{2}-t_{1}\right|^{\mu_{2}}+\|x-y\|_{\alpha}\right)
$$

for each $t_{1}, t_{2} \in \mathbb{R}$ and $x, y \in X_{\alpha}$;

(H6) $C M_{\alpha}\left(2 L_{1}+L_{2}\right) \frac{\omega^{1-\alpha}}{1-\alpha}+C_{1-\alpha} L_{2}<1$, where $C=\left\|(I-T(\omega))^{-1}\right\|$, then Eq. (1.1) has an $\omega$-periodic classical solution.

Proof Let $Q$ be the operator defined by (3.3) in the proof of Theorem 3.1. By the assumptions of Theorem 4.1 and the proof of Theorem 3.1, we know that the operator $Q: C_{\omega}\left(\mathbb{R}, X_{\alpha}\right) \rightarrow C_{\omega}\left(\mathbb{R}, X_{\alpha}\right)$ is well defined. From conditions (H4) and (H5), for any $u_{1}, u_{2} \in C_{\omega}\left(\mathbb{R}, X_{\alpha}\right), t \in \mathbb{R}$, similar to the proof of Theorem 3.3, we have

$$
\left\|Q u_{2}(t)-Q u_{1}(t)\right\|_{\alpha} \leq\left(C M_{\alpha}\left(2 L_{1}+L_{2}\right) \frac{\omega^{1-\alpha}}{1-\alpha}+C_{1-\alpha} L_{2}\right) \cdot\left\|u_{2}-u_{1}\right\|_{C_{\alpha}}
$$

which implies that

$$
\left\|Q u_{2}-Q u_{1}\right\|_{C \alpha} \leq\left(C M_{\alpha}\left(2 L_{1}+L_{2}\right) \frac{\omega^{1-\alpha}}{1-\alpha}+C_{1-\alpha} L_{2}\right) \cdot\left\|u_{2}-u_{1}\right\|_{C_{\alpha}}<\left\|u_{2}-u_{1}\right\|_{C_{\alpha}} .
$$

Hence, $Q: C_{\omega}\left(\mathbb{R}, X_{\alpha}\right) \rightarrow C_{\omega}\left(\mathbb{R}, X_{\alpha}\right)$ is a contraction, thus $Q$ has a unique fixed point $u_{0} \in$ $C_{\omega}\left(\mathbb{R}, X_{\alpha}\right)$. By the definition of $Q, u_{0}$ is an $\omega$-periodic mild solution of Eq. (1.1).

Next, we prove that $u_{0}$ is an $\omega$-periodic classical solution. From the periodicity of $u_{0}$, we only need prove it on $[0, \omega]$. Let $t \in[0, \omega]$ and $h(t)=F(t, u(t), u(t-\tau))-A G(t, u(t-\delta))$, then $h \in C([0, \omega], X)$. For $\forall \varepsilon \in(0, \omega)$, since $u_{0}$ is the $\omega$-periodic mild solution of Eq. (1.1), hence $u_{0}$ is the mild solution of the initial value problem

$$
\left\{\begin{array}{l}
(u(t)-G(t, u(t-\delta)))^{\prime}+A(u(t)-G(t, u(t-\delta)))=h(t), \quad t \in[\varepsilon, \omega], \\
u(\varepsilon)=u_{0}(\varepsilon) .
\end{array}\right.
$$


While $u_{0}(\varepsilon) \in X_{\alpha}$, from Lemma 2.4 it follows that

$$
u_{0} \in C^{\mu_{3}}\left([\varepsilon, \omega], X_{\alpha-\mu_{3}}\right) \hookrightarrow C^{\mu_{3}}([\varepsilon, \omega], X), \quad \mu_{3} \in(0, \alpha)
$$

On the other hand, from conditions (H4) and (H5), we can deduce $h \in C^{\mu}([\varepsilon, \omega], X)$, where $\mu=\min \left\{\mu_{1}, \mu_{2}, \mu_{3}\right\}$. By Lemma 2.5, we obtain that $u_{0}$ is a classical solution of Eq. (4.2) and satisfies

$$
u_{0} \in C^{1}((\varepsilon, \omega], X) \cap C\left([\varepsilon, \omega], X_{1}\right) .
$$

By the arbitrariness of $\varepsilon$, we claim that

$$
u_{0} \in C^{1}([0, \omega], X) \cap C\left([0, \omega], X_{1}\right) .
$$

Therefore, $u_{0}$ is an $\omega$-periodic classical solution of Eq. (1.1) and satisfies

$$
u_{0} \in C_{\omega}^{1}(\mathbb{R}, X) \cap C_{\omega}\left(\mathbb{R}, X_{1}\right) .
$$

The proof is completed.

Theorem 4.2 Assume that $X$ is a reflexive Banach space. For $\alpha \in[0,1)$, we assume that $G: \mathbb{R} \times X_{\alpha} \rightarrow X_{1}$ and $F: \mathbb{R} \times X_{\alpha}^{2} \rightarrow X$ are continuous functions, and for every $x, x_{0}, x_{1} \in X_{\alpha}$, $G(t, x), F\left(t, x_{0}, x_{1}\right)$ are $\omega$-periodic in $t$. If the conditions

$\left(\mathrm{H} 4^{\prime}\right)$ There exists a constant $L_{1}>0$ such that

$$
\left\|F\left(t_{2}, x_{0}, x_{1}\right)-F\left(t_{1}, y_{0}, y_{1}\right)\right\| \leq L_{1}\left(\left|t_{2}-t_{1}\right|+\left\|x_{0}-y_{0}\right\|_{\alpha}+\left\|x_{1}-y_{1}\right\|_{\alpha}\right)
$$

for any $t_{1}, t_{2} \in \mathbb{R}$ and $x_{0}, x_{1}, y_{0}, y_{1} \in X_{\alpha}$,

$\left(\mathrm{H} 5^{\prime}\right) G(t, \theta)=\theta$ for $t \in \mathbb{R}$, there exists $L_{2}$ such that

$$
\left\|A G\left(t_{2}, x\right)-A G\left(t_{1}, y\right)\right\| \leq L_{2}\left(\left|t_{2}-t_{1}\right|+\|x-y\|_{\alpha}\right)
$$

for each $t_{1}, t_{2} \in \mathbb{R}$ and $x, y \in X_{\alpha}$, and (H6) hold, then Eq. (1.1) has an w-periodic strong solution $u$.

Proof Let $Q$ be the operator defined by (3.3) in the proof of Theorem 3.1. For a given $r>0$, let $\bar{\Omega}_{r} \subset C_{\omega}\left(\mathbb{R}, X_{\alpha}\right)$ be defined by (3.2). By conditions ( $\left.\mathrm{H} 4^{\prime}\right)-\left(\mathrm{H} 6^{\prime}\right)$, one can use the same argument as in the proof of Theorem 3.1 to obtain that $\left(Q \bar{\Omega}_{r}\right) \subset \bar{\Omega}_{r}$.

For this $r$, consider the set

$$
\bar{\Omega}=\left\{u \in C_{\omega}\left(\mathbb{R}, X_{\alpha}\right)\left|\|u\|_{C \alpha} \leq r,\left\|u\left(t_{1}\right)-u\left(t_{2}\right)\right\|_{\alpha}<L^{*}\right| t_{2}-t_{1} \mid, t_{1}, t_{2} \in \mathbb{R}\right\}
$$

for some $L^{*}$ large enough. It is clear that $\bar{\Omega}$ is a convex closed and nonempty set. We shall prove that $Q$ has a fixed point on $\bar{\Omega}$. Obviously, from the proof of Theorem 3.1, it is sufficient to show that, for any $u \in \bar{\Omega}$,

$$
\left\|(Q u)\left(t_{2}\right)-(Q u)\left(t_{1}\right)\right\|_{\alpha} \leq L^{*}\left|t_{2}-t_{1}\right|, \quad \forall t_{1}, t_{2} \in \mathbb{R} .
$$


In fact, by the definition of $Q$, conditions $\left(\mathrm{H} 4^{\prime}\right),\left(\mathrm{H} 5^{\prime}\right)$, and (4.3), we have

$$
\begin{aligned}
& \left\|Q u\left(t_{2}\right)-Q u\left(t_{1}\right)\right\|_{\alpha} \\
& \leq \|(I-T(\omega))^{-1} \int_{t_{2}-\omega}^{t_{2}} T\left(t_{2}-s\right) F(s, u(s), u(s-\tau)) d s \\
& \left.-(I-T(\omega))^{-1} \int_{t_{1}-\omega}^{t_{1}} T\left(t_{1}-s\right) F(s, u(s), u(s-\tau))\right) d s \|_{\alpha} \\
& +\left\|G\left(t_{2}, u\left(t_{2}-\xi\right)\right)-G\left(t_{1}, u\left(t_{1}-\xi\right)\right)\right\|_{\alpha} \\
& +\|(I-T(\omega))^{-1} \int_{t_{2}-\omega}^{t_{2}} T\left(t_{2}-s\right) A G(s, u(s-\xi)) d s \\
& -(I-T(\omega))^{-1} \int_{t_{1}-\omega}^{t_{1}} T\left(t_{1}-s\right) A G(s, u(s-\xi)) d s \|_{\alpha} \\
& \leq \|(I-T(\omega))^{-1} \int_{0}^{\omega} A^{\alpha} T(s)\left(F\left(t_{2}-s, u\left(t_{2}-s\right), u\left(t_{2}-s-\tau\right)\right)\right. \\
& \left.-F\left(t_{1}-s, u\left(t_{1}-s\right), u\left(t_{1}-s-\tau\right)\right)\right) d s \| \\
& +C_{1-\alpha} \| A G\left(t_{2}, u\left(t_{2}-\xi\right)\right)-A G\left(t_{1}, u\left(t_{1}-\xi\right) \|\right. \\
& +\|(I-T(\omega))^{-1} \int_{0}^{\omega} A^{\alpha} T(s)\left(A G\left(t_{2}-s, u\left(t_{2}-s-\xi\right)\right)\right. \\
& \left.-A G\left(t_{1}-s, u\left(t_{2}-s-\xi\right)\right)\right) d s \| \\
& \leq C M_{\alpha} \frac{\omega^{1-\alpha}}{1-\alpha} L_{1}\left(1+2 L^{*}\right)\left|t_{2}-t_{1}\right|+C_{1-\alpha} L_{2}\left(1+L^{*}\right)\left|t_{2}-t_{1}\right| \\
& +C M_{\alpha} \frac{\omega^{1-\alpha}}{1-\alpha} L_{2}\left(1+L^{*}\right)\left|t_{2}-t_{1}\right| \\
& =\left(C M_{\alpha} \frac{\omega^{1-\alpha}}{1-\alpha}\left(L_{1}+L_{2}\right)+C_{1-\alpha} L_{2}+\left(C M_{\alpha}\left(2 L_{1}+L_{2}\right) \frac{\omega^{1-\alpha}}{1-\alpha}+C_{1-\alpha} L_{2}\right) L^{*}\right)\left|t_{2}-t_{1}\right| \\
& :=\left(K_{0}+K^{*} L^{*}\right)\left|t_{2}-t_{1}\right|
\end{aligned}
$$

where $K_{0}=C M_{\alpha} \frac{\omega^{1-\alpha}}{1-\alpha}\left(L_{1}+L_{2}\right)+C_{1-\alpha} L_{2}$ is a constant independent of $L^{*}$, and $K^{*}=$ $\left(C M_{\alpha}\left(2 L_{1}+L_{2}\right) \frac{\omega^{1-\alpha}}{1-\alpha}+C_{1-\alpha} L_{2}\right)<1$. Hence,

$$
\left\|Q u\left(t_{1}\right)-Q u\left(t_{1}\right)\right\|_{\alpha} \leq L^{*}\left|t_{2}-t_{1}\right| \quad \text { for all } t_{2}, t_{1} \in \mathbb{R}
$$

whenever $L^{*} \geq \frac{K_{0}}{1-K^{*}}$. Therefore, $Q$ has a fixed point $u$ which is an $\omega$-periodic mild solution of Eq. (1.1).

By the above calculation, we see that, for this $u(\cdot)$, all the following functions:

$$
\begin{aligned}
& g(t)=G(t, u(t-\xi)), \\
& \Phi(t)=(I-T(\omega))^{-1} \int_{t-\omega}^{t} T(t-s) F(s, u(s), u(s-\tau)) d s, \\
& \Psi(t)=(I-T(\omega))^{-1} \int_{t-\omega}^{t} T(t-s) A G(s, u(x-\xi)) d s
\end{aligned}
$$


are Lipschitz continuous, respectively. Since the $u$ is Lipschitz continuous on $\mathbb{R}$ and the space $X_{\alpha}$ is reflexive by the assumption and Lemma 2.3, then a result of [27] asserts that $u(\cdot)$ is a.e. differentiable on $\mathbb{R}$ and $u^{\prime}(\cdot) \in L_{\text {loc }}^{1}\left(\mathbb{R}, X_{\alpha}\right)$. Furthermore, by a standard argument as Theorem 4.2.4 in [38], we can obtain that

$$
\begin{aligned}
\Phi^{\prime}(t)= & (I-T(\omega))^{-1}((I-T(\omega)) F(t, u(t), u(t-\tau)) \\
& \left.-\int_{t-\omega}^{t} A T(t-s) F(s, u(s), u(s-\tau)) d s\right) \\
\Psi^{\prime}(t)= & (I-T(\omega))^{-1}((I-T(\omega)) A G(t, u(t-\xi)) \\
& \left.-\int_{t-\omega}^{t} A T(t-s) A G(s, u(x-\xi)) d s\right) .
\end{aligned}
$$

Hence, for almost every $t \in \mathbb{R}$,

$$
\begin{aligned}
u^{\prime}(t)= & \Phi^{\prime}(t)+g^{\prime}(t)-\Psi^{\prime}(t) \\
= & (I-T(\omega))^{-1}((I-T(\omega)) F(t, u(t), u(t-\tau)) \\
& \left.-\int_{t-\omega}^{t} A T(t-s) F(s, u(s), u(s-\tau)) d s\right)+G^{\prime}(t, u(t-\xi)) \\
& -(I-T(\omega))^{-1}((I-T(\omega)) A G(t, u(t-\xi)) \\
& \left.-\int_{t-\omega}^{t} A T(t-s) A G(s, u(x-\xi)) d s\right) \\
= & F(t, u(t), u(t-\tau))-A G(t, u(t-\xi))+G^{\prime}(t, u(t-\xi)) \\
& -A\left((I-T(\omega))^{-1} \int_{t-\omega}^{t} T(t-s) F(s, u(s), u(s-\tau)) d s\right. \\
& \left.-(I-T(\omega))^{-1} \int_{t-\omega}^{t} T(t-s) A G(s, u(x-\xi)) d s\right) \\
= & F(t, u(t), u(t-\tau))-A G(t, u(t-\xi))+G^{\prime}(t, u(t-\xi)) \\
& -A(u(t)-G(t, u(t-\xi))),
\end{aligned}
$$

which implies that

$$
(u(t)-G(t, u(t-\xi)))^{\prime}+A u(t)=F(t, u(t), u(t-\tau)), \quad \text { a.e } t \in \mathbb{R} .
$$

This shows that $u$ is a strong solution for Eq. (1.1) and the proof is completed.

\section{Application}

In this section, we present one example, which indicates how our abstract results can be applied to concrete problems. 
Consider the following parabolic boundary value problem with delays:

$$
\left\{\begin{array}{l}
\frac{\partial}{\partial t}\left(u(x, t)-\int_{0}^{1} g(x, y, t, u(y, t-\xi)) d y\right)-\frac{\partial^{2}}{\partial x^{2}} u(x, t) \\
\quad=f(x, t, u(x, t), u(x, t-\tau)), \quad x \in[0,1], t \in \mathbb{R}, \\
u(0, t)=u(1, t)=0,
\end{array}\right.
$$

where $g \in C([0,1] \times[0,1] \times \mathbb{R} \times \mathbb{R}, \mathbb{R}), f \in C([0,1] \times \mathbb{R} \times \mathbb{R} \times \mathbb{R}, \mathbb{R})$, and $f, g$ are $\omega$-periodic in $t$, and $\xi, \tau$ are positive constants which denote the time delays.

To treat this system in the abstract form (1.1), we choose the space $X=L^{2}([0,1], \mathbb{R})$, endowed with the $L^{2}$-norm $\|\cdot\|_{L^{2}}$. We remark that $X$ is reflexive.

Define operator $A: D(A) \subset X \rightarrow X$ by

$$
D(A):=\left\{u \in X \mid u^{\prime \prime}, u^{\prime} \in X, u(0)=u(1)=0\right\}, \quad A u=-\frac{\partial^{2} u}{\partial x^{2}} .
$$

It is well know that $-A$ generates an exponentially stable compact analytic semigroup $T(t)$ $(t \geq 0)$ in $X$. In addition, we note that $0 \in \rho(A)$ and that fractional powers of $A$ are well defined. Moveover, $A$ has a discrete spectrum with eigenvalues of the form $n^{2} \pi^{2}, n \in \mathbb{N}$, and the associated normalized eigenfunctions are given by $e_{n}(x)=\sqrt{2} \sin (n \pi x)$ for $x \in$ $[0,1]$, the associated semigroup $T(t)(t \geq 0)$ is explicitly given by

$$
T(t) u=\sum_{n=1}^{\infty} e^{-n^{2} \pi^{2} t}\left(u, e_{n}\right) e_{n}, \quad t \geq 0, u \in X,
$$

where $(\cdot, \cdot)$ is an inner product on $X$, and it is not difficult to verify that $\|T(t)\| \leq e^{-\pi^{2} t}$ for all $t \geq 0$. Hence, we take $M=1, M_{\frac{1}{2}}=\Gamma\left(\frac{1}{2}\right)$ and $\left\|(I-T(\omega))^{-1}\right\| \leq \frac{1}{1-e^{-\pi^{2} \omega}}$. The proof of the following lemma can be found in [40].

Lemma 5.1 If $v \in D\left(A^{\frac{1}{2}}\right)$, then $v$ is absolutely continuous with $v^{\prime} \in X$ and $\left\|v^{\prime}\right\|_{L^{2}}=\left\|A^{\frac{1}{2}} v\right\|_{L^{2}}$.

According to Lemma 5.1, we define the Banach space $\left.X_{\frac{1}{2}}:=\left(D^{\frac{1}{2}}\right),\|\cdot\|_{\frac{1}{2}}\right)$, where $\|v\|_{\frac{1}{2}}=\left\|A^{\frac{1}{2}} v\right\|_{L^{2}}$ for all $v \in X_{\frac{1}{2}}$. Under the above assumptions we discuss the existence and regularity of mild solutions of time $\omega$-periodic solutions of the delays parabolic boundary value problem (5.1).

Proposition 5.1 If the following conditions hold:

(F1) There are positive constants $a_{0}, a_{1}$, and $K$ such that, for every $(x, t, v, w) \in[0,1] \times \mathbb{R} \times \mathbb{R} \times \mathbb{R}$,

$$
|f(x, t, v, w)| \leq a_{0}|v|+a_{1}|w|+K
$$

(F2) $g:[0,1] \times[0,1] \times \mathbb{R} \times \mathbb{R} \rightarrow \mathbb{R}$ satisfies the following conditions:

(i) There is a positive function $b:[0,1] \times[0,1] \times \mathbb{R} \rightarrow \mathbb{R}^{+}$such that

$$
\left|g\left(x, y, t, v_{2}\right)-g\left(x, y, t, v_{1}\right)\right| \leq b(x, y, t)\left|v_{2}-v_{1}\right|
$$


for all $x, y \in[0,1], t \in \mathbb{R}$ and $v_{1}, v_{2} \in \mathbb{R} ;$ moreover, $(x, y, t) \mapsto \frac{\partial^{2}}{\partial x^{2}} b(x, y, t)$ is well defined and measurable with

$$
l^{2}:=\max _{t \in[0, \omega]} \int_{0}^{1} \int_{0}^{1}\left(\frac{\partial^{2}}{\partial x^{2}} b(x, y, t)\right)^{2} d y d x<\infty,
$$

(ii) $g(0, y, t, v)=g(1, y, t, v)=0$ for all $t \in \mathbb{R}, y \in[0,1]$ and $v \in \mathbb{R}$;

(F3) $\frac{2 \omega^{\frac{1}{2}}}{1-e^{-\pi^{2} \omega}} \Gamma\left(\frac{1}{2}\right)\left(a_{0}+a_{1}+l\right)+l<1$, then the neutral partial differential equation with delays (5.1) has at least one time $\omega$-periodic mild solution.

Proof Let $F: \mathbb{R} \times X_{\frac{1}{2}} \times X_{\frac{1}{2}} \rightarrow X$ and $G: \mathbb{R} \times X_{\frac{1}{2}} \rightarrow X$ be defined by

$$
\begin{aligned}
& F(t, \phi, \varphi)(x)=f(x, t, \phi(x), \varphi(x)), \\
& G(t, \phi)(x)=\int_{0}^{1} g(x, y, t, \phi(y)) d y
\end{aligned}
$$

for $t \in \mathbb{R}, \phi, \varphi \in X_{\frac{1}{2}}$ and $x \in[0,1]$. Moreover, if $u: \mathbb{R} \times[0,1] \rightarrow \mathbb{R}$, we define $u: \mathbb{R} \rightarrow X$ by $u(t)(x)=u(x, t)$ for $x \in[0,1]$, which implies that $u(t-\tau)(\cdot)=u(\cdot, t-\tau)$ and $u(t-\xi)(\cdot)=$ $u(\cdot, t-\xi)$. Therefore, the partial differential equation with delays (5.1) can be rewritten into the abstract evolution equation with delays (1.1).

From the definition of $F$ and assumption (F1), we can get $F: \mathbb{R} \times X_{\frac{1}{2}} \times X_{\frac{1}{2}} \rightarrow X$ is a continuous function, and for each $\phi, \varphi \in X_{\frac{1}{2}}$ and $t \in \mathbb{R}$,

$$
\begin{aligned}
\|F(t, \phi, \varphi)\|_{L^{2}} & =\left(\int_{0}^{1}(f(x, t, \phi(x), \varphi(x)))^{2} d x\right)^{\frac{1}{2}} \\
& \leq\left(\int_{0}^{1}\left(a_{0}|\phi(x)|+a_{1}|\varphi(x)|+K\right)^{2} d x\right)^{\frac{1}{2}} \\
& \leq a_{0}\|\phi\|_{L^{2}}+a_{1}\|\varphi\|_{L^{2}}+K \\
& \leq a_{0}\|\phi\|_{\frac{1}{2}}+a_{1}\|\varphi\|_{\frac{1}{2}}+K
\end{aligned}
$$

thus, condition ( $\left.\mathrm{H} 1^{\prime}\right)$ in Sect. 3 holds.

By the definition of $G$ and assumption (F2), we see that $G: \mathbb{R} \times X_{\frac{1}{2}} \rightarrow X_{1}\left(X_{1}:=D(A)\right)$ is continuous and

$$
\begin{aligned}
& \|A G(t, \phi)-A G(t, \varphi)\|_{L^{2}}^{2} \\
& \quad \leq \int_{0}^{1} \int_{0}^{1}\left(\frac{\partial^{2}}{\partial x^{2}} b(x, y, t) \cdot|\phi(y)-\varphi(y)|\right)^{2} d y d x \\
& \quad \leq \int_{0}^{1} \int_{0}^{1}\left(\frac{\partial^{2}}{\partial x^{2}} b(x, y, t)\right)^{2} d y d x \cdot\|\phi-\varphi\|_{L^{2}}^{2} \\
& \quad \leq l^{2}\|\phi-\varphi\|_{\frac{1}{2}}^{2},
\end{aligned}
$$

for each $t \in \mathbb{R}$ and $\phi, \varphi \in X_{\frac{1}{2}}$. Thus, condition (H2) in Sect. 3 holds.

Finally, by (F3) and Corollary 3.2, it follows that the neutral partial differential equation with delays (5.1) has at least one time $\omega$-periodic mild solution. The proof is completed. 
For showing the existence of classical and strong solutions, the following assumptions are needed:

(F4) There exist constants $l_{1}$ and $\mu_{1} \in(0,1]$ such that, for every $t_{i}, v_{i}, w_{i} \in \mathbb{R}(i=1,2)$ and $x \in[0,1]$,

$$
\left|f\left(x, t_{2}, v_{2}, w_{2}\right)-f\left(x, t_{1}, v_{1}, w_{1}\right)\right| \leq l_{1}\left(\left|t_{2}-t_{1}\right|^{\mu_{1}}+\left|v_{2}-v_{1}\right|+\left|w_{2}-w_{1}\right|\right),
$$

(F5) $g:[0,1] \times[0,1] \times \mathbb{R} \times \mathbb{R} \rightarrow \mathbb{R}$ satisfies the following conditions:

(i) $(x, y, t, v) \mapsto \frac{\partial^{2}}{\partial x^{2}} g(x, y, t, v)$ is well defined and measurable. Moreover, there are constants $l_{2}>0$ and $\mu_{2} \in(0,1]$ such that, for every $t_{i}, v_{i} \in \mathbb{R}(i=1,2)$,

$$
\left|\int_{0}^{1} \frac{\partial^{2}}{\partial x^{2}} g\left(x, y, t_{2}, v_{2}\right) d y-\int_{0}^{1} \frac{\partial^{2}}{\partial x^{2}} g\left(x, y, t_{1}, v_{1}\right) d y\right| \leq l_{2}\left(\left|t_{2}-t_{1}\right|^{\mu_{2}}+\left|v_{2}-v_{1}\right|\right),
$$

(ii) $g(0, y, t, v)=g(1, y, t, v)=0$ for all $t \in \mathbb{R}, y \in[0,1]$ and $v \in \mathbb{R}$,

(F6) $\frac{2 \omega^{\frac{1}{2}}}{1-e^{-\pi^{2} \omega}} \Gamma\left(\frac{1}{2}\right)\left(2 l_{1}+l_{2}\right)+l_{2}<1$.

Hence, for every $t_{1}, t_{2} \in \mathbb{R}$ and $\phi_{1}, \varphi_{1}, \phi_{2}, \varphi_{2} \in X_{\frac{1}{2}}$, we have

$$
\begin{aligned}
\| & F\left(t_{2}, \phi_{2}, \varphi_{2}\right)-F\left(t_{1}, \phi_{1}, \varphi_{1}\right) \|_{L^{2}} \\
& =\left(\int_{0}^{1}\left(f\left(x, t_{2}, \phi_{2}(x), \varphi_{2}(x)\right)-f\left(x, t_{1}, \phi_{1}(x), \varphi_{1}(x)\right)\right)^{2} d x\right)^{\frac{1}{2}} \\
& \leq\left(\int_{0}^{1} l^{2}\left(\left|t_{2}-t_{1}\right|^{\mu_{1}}+\left|\phi_{2}(x)-\phi_{1}(x)\right|+\left|\varphi_{2}(x)-\varphi_{1}(x)\right|\right)^{2} d x\right)^{\frac{1}{2}} \\
& \leq l_{1}\left(\left|t_{2}-t_{1}\right|^{\mu_{1}}+\left\|\phi_{2}-\phi_{1}\right\|_{L^{2}}+\left\|\varphi_{2}-\varphi_{1}\right\|_{L^{2}}\right) \\
& \leq l_{1}\left(\left|t_{2}-t_{1}\right|^{\mu_{1}}+\left\|\left(\phi_{2}-\phi_{1}\right)\right\|_{\frac{1}{2}}+\left\|\varphi_{2}-\varphi_{1}\right\|_{\frac{1}{2}}\right),
\end{aligned}
$$

and

$$
\begin{aligned}
\left\|A G\left(t_{2}, \phi_{2}\right)-A G\left(t_{1}, \phi_{1}\right)\right\|_{L^{2}} \\
\quad=\left(\int_{0}^{1}\left(\int_{0}^{1} \frac{\partial^{2}}{\partial x^{2}} g\left(x, y, t_{2}, \phi_{2}(y)\right) d y-\int_{0}^{1} \frac{\partial^{2}}{\partial x^{2}} g\left(x, y, t_{1}, \phi_{1}(y)\right) d y\right)^{2} d x\right)^{\frac{1}{2}} \\
\quad \leq\left(\int_{0}^{1} l_{2}^{2}\left(\left|t_{2}-t_{1}\right|^{\mu_{2}}+\left|\phi_{2}(x)-\phi_{1}(x)\right|\right)^{2} d x\right)^{\frac{1}{2}} \\
\leq l_{2}\left(\left|t_{2}-t_{1}\right|^{\mu_{2}}+\left\|\phi_{2}-\phi_{1}\right\|_{L^{2}}\right) \\
\leq l_{2}\left(\left|t_{2}-t_{1}\right|^{\mu_{2}}+\left\|\phi_{2}-\phi_{1}\right\|_{\frac{1}{2}}\right),
\end{aligned}
$$

which implies that conditions ( $\mathrm{H} 4)$ and $(\mathrm{H} 5)$ for $\mu_{1}, \mu_{2} \in(0,1)$ (or $\left(\mathrm{H} 4^{\prime}\right)$ and $\left(\mathrm{H} 5^{\prime}\right)$ for $\mu_{1}=\mu_{2}=1$ ) hold. On the other hand, by condition (F6), we can easily prove that condition (H6) holds in Sect. 4.

Consequently, all the conditions stated in Theorem 4.1 and Theorem 4.2 are satisfied, and we obtain the following interesting results.

Proposition 5.2 If conditions (F4)-(F5) hold for $\mu_{1}, \mu_{2} \in(0,1)$, then the neutral partial differential equation with delays (5.1) has time $\omega$-periodic classical solutions. 
Proposition 5.3 If conditions (F4)-(F5) hold for $\mu_{1}=\mu_{2}=1$, then the neutral partial differential equation with delays (5.1) has time $\omega$-periodic strong solutions.

\author{
Acknowledgements \\ The authors are most grateful to the editor and anonymous referees for the careful reading of the manuscript and \\ valuable suggestions that helped in significantly improving an earlier version of this paper.
}

\title{
Funding
}

This work was supported by NNSF of China (11261053) and NSF of Gansu Province (1208RJZA129).

Availability of data and materials

Not applicable.

Competing interests

Q. Li and $\mathrm{H}$. Zhang declare that they have no competing interests.

Authors' contributions

$\mathrm{QL}$ and $\mathrm{HZ}$ contributed equally and significantly in writing this article. All authors read and approved the finial manuscript.

\section{Author details}

${ }^{1}$ Department of Mathematics, Shanxi Normal University, Linfen, People's Republic of China. ${ }^{2}$ Department of Mathematics, Northwest Minzui University, Lanzhou, People's Republic of China.

\section{Publisher's Note}

Springer Nature remains neutral with regard to jurisdictional claims in published maps and institutional affiliations.

Received: 20 January 2019 Accepted: 31 July 2019 Published online: 08 August 2019

\section{References}

1. Adimy, M., Bouzahir, H., Ezzinbi, K.: Existence and stability for some partial neutral functional differential equations with infinite delay. J. Math. Anal. Appl. 294, 438-461 (2004)

2. Adimy, M., Ezzinbi, K.: A class of linear partial neutral functional differential equations with nondense domain. J. Differ. Equ. 147, 285-332 (1998)

3. Adimy, M., Ezzinbi, K.: Existence and stability in the $\alpha$-norm for partial functional equations of neutral type. Ann. Mat. Pura Appl. 185, 437-460 (2006)

4. Babram, M.A., Ezzinbi, K.: Periodic solutions of functional differential equations of neutral type. J. Math. Anal. Appl. 204, 898-909 (1996)

5. Benkhalti, R., Elazzouzi, A., Ezzinbi, K.: Periodic solutions for some partial neutral functional differential equations. Electron. J. Differ. Equ. 2006, 56 (2006)

6. Benkhalti, R., Elazzouzi, A., Ezzinbi, K.: Periodic solutions for some nonlinear partial neutral functional differential equations. Int. J. Bifurc. Chaos Appl. Sci. Eng. 20, 545-555 (2010)

7. Benkhalti, R., Ezzinbi, K.: Periodic solutions for some partial functional differential equations. J. Appl. Math. Stoch. Anal. 1, 9-18 (2004)

8. Burton, T.A., Zhang, B.: Periodic solutions of abstract differential equations with infinite delay. J. Differ. Equ. 90 357-396 (1991)

9. Cannarsa, P., Sforza, D.: Global solutions of abstract semilinear parabolic equations with memory terms. NoDEA Nonlinear Differ. Equ. Appl. 10, 399-430 (2003)

10. Chang, J., Liu, H.: Existence of solutions for a class of neutral partial differential equations with nonlocal conditions in the $\alpha$-norm. Nonlinear Anal. 71, 3759-3768 (2009)

11. Chen, Y.: The existence of periodic solutions for a class of neutral differential difference equations. J. Aust. Math. Soc Ser. B 33, 507-516 (1992)

12. Ezzinbi, K., Fu, X.L.: Existence and regularity of solutions for some neutral partial differential equations with non-local conditions. Nonlinear Anal. 57, 1029-1041 (2004)

13. Ezzinbi, K., Ghnimib, S.: Existence and regularity of solutions for neutral partial functional integrodifferential equations. Nonlinear Anal., Real World Appl. 11, 2335-2344 (2010)

14. Ezzinbi, K., Kyelem, B.A., Ouaro, S.: Periodicity in the $\alpha$-norm for partial functional differential equations in fading memory spaces. Nonlinear Anal. 97, 30-54 (2014)

15. Ezzinbi, K., Lui, J.: Periodic solutions of non-densely defined delay evolutions equations. J. Appl. Math. Stoch. Anal. 15, $113-123(2002)$

16. Fu, X.: Existence of solutions and periodic solutions for abstract neutral equations with unbounded delay. Dyn. Contin. Discrete Impuls. Syst., Ser. A Math. Anal. 15, 17-35 (2008)

17. Fu, X., Liu, X.: Existence of periodic solutions for abstract neutral non-autonomous equations with infinite delay. J. Math. Anal. Appl. 325, 249-267 (2007)

18. Hale, J.: Partial neutral functional-differential equations. Rev. Roum. Math. Pures Appl. 39, 339-344 (1994)

19. Hale, J.K., Verduyn Lunel, S.M.: Introduction to Functional Differential Equations. Springer, Berlin (1993)

20. Hernández, E.: Existence results for partial neutral integrodifferential equations with unbounded delay. J. Math. Anal. Appl. 292, 194-210 (2004)

21. Hernández, E., Henriquez, H.R.: Existence of periodic solutions of partial neutral functional differential equations with unbounded delay. J. Math. Anal. Appl. 221, 499-522 (1998) 
22. Hernández, E., O’Regan, D., Ponce, R.: On $C^{\alpha}$-Hölder classical solutions for non-autonomous neutral differential equations: the nonlinear case. J. Math. Anal. Appl. 420, 1814-1831 (2014)

23. Hernández, E., Pelicer, M.L.: Asymptotically almost periodic and almost periodic solutions for partial neutral differential equations. Appl. Math. Lett. 18, 1265-1272 (2005)

24. Hernández, E., Pierri, M., Prokopczyk, A.: On a class of abstract neutral functional differential equations. Nonlinear Anal. 74, 3633-3643 (2011)

25. Hino, Y., Murakami, S.: Periodic solutions of linear Volterra systems in differential equations. In: Lecture Notes in Pure and App. Math., vol. 118, pp. 319-326. Dekker, New York (1987)

26. Huy, N., Dang, N.: Dichotomy and periodic solutions to partial functional differential equations. Discrete Contin. Dyn. Syst., Ser. B 22, 3127-3144 (2017)

27. Komura, J.: Differentiability of nonlinear semigroups. J. Math. Soc. Jpn. 21, 375-402 (1969)

28. Li, Q., Li, Y.: Existence of positive periodic solutions for abstract evolution equations. Adv. Differ. Equ. $2015,135(2015)$

29. Li, Y.: Existence and uniqueness of positive periodic solution for abstract semilinear evolution equations. J. Syst. Sci. Math. Sci. 25, 720-728 (2005) (in Chinese)

30. Li, Y.: Existence and asymptotic stability of periodic solution for evolution equations with delays. J. Funct. Anal. 261, 1309-1324 (2011)

31. Liang, J., et al.: Periodicity of solutions to the Cauchy problem for nonautonomous impulsive delay evolution equations in Banach spaces. Anal. Appl. 15, 457-476 (2017)

32. Liang, J., Liu, J.H., Xiao, T.J.: Condensing operators and periodic solutions of infinite delay impulsive evolution equations. Discrete Contin. Dyn. Syst., Ser. B 10,475-485 (2017)

33. Liu, H., Chang, J.: Existence for a class of partial differential equations with nonlocal conditions. Nonlinear Anal. 70 3076-3083 (2009)

34. Liu, J.: Bounded and periodic solutions of finite delays evolution equations. Nonlinear Anal. 34, 101-111 (1998)

35. Liu, J.: Periodic solutions of infinite delay evolution equations. J. Math. Anal. Appl. 247, 644-727 (2000)

36. Liu, J.: Bounded and periodic solutions of infinite delay evolution equations. J. Math. Anal. Appl. 286, 705-712 (2003)

37. Lunardi, A.: On the linear heat equation with fading memory. SIAM J. Math. Anal. 21, 1213-1224 (1990)

38. Pazy, A.: Semigroup of Linear Operators and Applications to Partial Differential Equations. Springer, New York (1993)

39. Sadovskii, B.N.: On a fixed point principle. Funct. Anal. Appl. 1, 74-76 (1967)

40. Travis, C.C., Webb, G.F.: Existence, stability and compactness with $\alpha$-norm for partial functional differential equations. Transl. Am. Math. Soc. 240, 129-143 (1978)

41. Triggiani, R.: On the stabilizability problem in Banach space. J. Math. Anal. Appl. 52, 383-403 (1975)

42. Wu, J.: Theory and Application of Partial Functional Differential Equations. Springer, New York (1996)

43. $W u, J ., X i a, H .:$ The existence of periodic solutions to integro-differential equations of neutral type via limiting equations. Math. Proc. Camb. Philos. Soc. 112, 403-418 (1992)

44. Xiang, X., Ahmed, N.U.: Existence of periodic solutions of semilinear evolution equations with time lags. Nonlinear Anal. 18, 1063-1070 (1992)

\section{Submit your manuscript to a SpringerOpen ${ }^{\circ}$ journal and benefit from:}

- Convenient online submission

- Rigorous peer review

- Open access: articles freely available online

- High visibility within the field

- Retaining the copyright to your article

Submit your next manuscript at $\boldsymbol{s p r i n g e r o p e n . c o m ~}$ 